\title{
More-Stable EPLL
}

\author{
Saeed Golestan ${ }^{\circledR}$, Senior Member, IEEE, Jose Matas ${ }^{\circledR}$, Abdullah M. Abusorrah ${ }^{\circledR}$, Senior Member, IEEE, \\ and Josep M. Guerrero ${ }^{\circledR}$, Fellow, IEEE
}

\begin{abstract}
The enhanced phase-locked loop (EPLL) is one of the most famous PLLs in single-phase applications and a versatile tool for different signal processing applications, especially for the grid synchronization of power converters. Recently, it has been proved that the EPLL has a quite narrow stable zone (compared to its unstable zone) in the positive parameter space from a small-signal point of view. This zone will be even more narrow if maintaining a minimum stability margin is required. This article aims to modify the EPLL structure to improve its stability margin, and at the same time, make it unconditionally stable in the positive parameter space from a small-signal point of view.
\end{abstract}

Index Terms-Enhanced phase-locked loop (EPLL), singlephase systems, synchronization.

\section{INTRODUCTION}

$\mathbf{T}$ HE enhanced phase-locked loop (EPLL) is one of the most famous PLLs in single-phase applications. The block diagram representation of this PLL can be observed in Fig. 1 [1], in which $v$ is the single-phase input signal, $\hat{V}_{1}, \hat{\theta}_{1}$, and $\hat{\omega}$ are estimates of the amplitude, phase angle, and angular frequency of this signal, respectively, and $k_{p}, k_{i}$, and $k_{v}$ are the control gains. ${ }^{1}$ From the structural point of view, the EPLL looks a bit different from a typical single-phase PLL, which is often based on a synchronous reference frame PLL (SRF-PLL) and a fictitious ( $\beta$-axis) signal generator [2]. However, mathematically speaking, it is equivalent to a single-phase SRF-PLL that generates its $\beta$-axis input signal directly using the estimated phase angle and amplitude [3].

The EPLL has received considerable research attention over the past two decades. A part of these research activities has

Manuscript received January 29, 2021; revised May 16, 2021; accepted June 24, 2021. Date of publication July 26, 2021; date of current version September 16,2021 . This work was supported in part by the Deanship of Scientific Research, King Abdulaziz University, Jeddah under Grant RG-22-135-41, and in part by VILLUM FONDEN under the VILLUM Investigator Grant 25920: Center for Research on Microgrids (CROM). Recommended for publication by Associate Editor L. Peng. (Corresponding author: Saeed Golestan.)

Saeed Golestan and Josep M. Guerrero are with the AAU Energy, Faculty of Engineering and Science, Aalborg University, 9220 Aalborg, Denmark (e-mail: s.golestan@ieee.org; joz@et.aau.dk).

Jose Matas is with the Electric Engineering Department, Polytechnic University of Catalonia (EEBE-UPC), 08019 Barcelona, Spain (e-mail: jose.matas@upc.edu).

Abdullah M. Abusorrah is with the Department of Electrical and Computer Engineering, Faculty of Engineering, and Center of Research Excellence in Renewable Energy and Power Systems, King Abdulaziz University, Jeddah 21481, Saudi Arabia (e-mail: aabusorrah@kau.edu.sa).

Color versions of one or more figures in this article are available at https: //doi.org/10.1109/TPEL.2021.3099839.

Digital Object Identifier 10.1109/TPEL.2021.3099839

${ }^{1}$ Throughout this article, $k_{p}=k_{v}$ is assumed. This selection is often considered as an optimal choice in the literature [1]. focused on applying the EPLL and its extended versions to different signal processing applications. For instance, in addition to the synchronization of power converters in both single-phase and three-phase applications, the EPLL and its extended versions have been widely employed for the detection and separation of fundamental and disturbance (dc, harmonics, and interharmonic) components of power signals [4], [5], computation of synchrophasors [6]-[8], fault analysis [9], estimation of parameters of electromagnetic oscillations [10], and implementations of robust resonant controllers [11], among other applications.

Improving the EPLL filtering capability and/or dynamic performance in the grid synchronization applications has also been a main focus of research in the past years. In [12], for instance, an adaptive method to improve the start-up and phase jump tracking ability of the EPLL without considerably affecting its filtering capability was proposed. In [13] and [14], including inloop filters and window functions into the EPLL control loops to provide a more smooth estimation of the grid voltage parameters under noisy conditions was introduced. In [5], adding a dc offset estimation/rejection loop to the EPLL to provide an estimation of the input dc component, and at the same time, make the EPLL immune to the disturbing effect of the dc offset was proposed. In [15], including a single nonadaptive bandpass filter before the EPLL input and a compensator in its output was proposed. The bandpass filter rejects the dc offset and attenuates the input harmonics, and the compensator corrects errors caused by the bandpass filter under off-nominal frequencies. In [16], including an adaptive filter before the EPLL input was proposed. This filter, which is based on delayed signal cancellation operators, improves the EPLL filtering capability without considerably affecting its dynamic response.

Despite the fact that the EPLL is structurally robust under extreme scenarios such as low sampling frequencies and fixedpoint implementations with a limited number of bits [10], [11], recently it has been proved that it has a quite narrow stable zone (compared to its unstable zone) in the positive parameter space from a small-signal point of view [17]. This stable zone becomes even more narrow if maintaining a minimum stability margin is required.

To deal with the aforementioned problem, a more-stable EPLL (MsEPLL), which is simply realized by adding some nonlinear terms to the traditional EPLL, is presented in this article. An analytic linear time-periodic (LTP) model for the MsEPLL is then obtained, and its stability is investigated. It will be shown that the MsEPLL has a more decent stability margin compared to the EPLL. It will also be demonstrated that the 


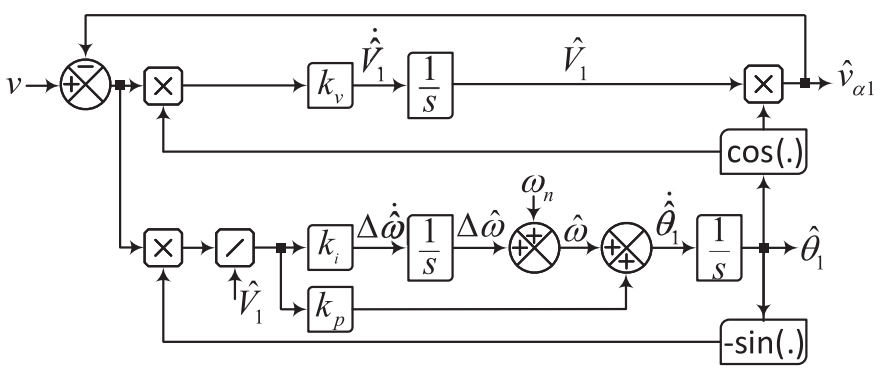

Fig. 1. Conventional EPLL.

MsEPLL, contrary to the EPLL, is unconditionally stable in the positive parameter space from a small-signal point of view.

\section{More-Stable EPLL (MsEPLL)}

Assume that the single-phase input signal of the EPLL is as (1), where $V_{1}$ and $\theta_{1}$ are the amplitude and phase angle of this signal, respectively

$$
v(t)=V_{1} \cos \left(\theta_{1}\right) .
$$

In this case, the governing nonlinear differential equations (GNDEs) of the EPLL can be simply obtained from Fig. 1 as

$$
\begin{aligned}
& \dot{\hat{\omega}}=\Delta \dot{\hat{\omega}}=\frac{k_{i}}{2 \hat{V}_{1}}\left[V_{1} \sin \left(\theta_{1}-\hat{\theta}_{1}\right)\right. \\
& \left.+\hat{\mathbf{V}}_{\mathbf{1}} \sin \left(\mathbf{2} \hat{\theta}_{\mathbf{1}}\right)-\mathbf{V}_{\mathbf{1}} \sin \left(\theta_{\mathbf{1}}+\hat{\theta}_{\mathbf{1}}\right)\right] \\
& \dot{\hat{\theta}}_{1}=\hat{\omega}+\frac{k_{p}}{k_{i}} \Delta \dot{\hat{\omega}} \\
& \dot{\hat{\mathbf{V}}}_{1}=\frac{k_{v}}{2}\left[V_{1} \cos \left(\theta_{1}-\hat{\theta}_{1}\right)-\hat{V}_{1}\right. \\
& \left.+\mathrm{V}_{1} \cos \left(\theta_{1}+\hat{\theta}_{1}\right)-\hat{\mathrm{V}}_{1} \cos \left(2 \hat{\theta}_{1}\right)\right] .
\end{aligned}
$$

The highlighted terms in (2) and (4) are some doublefrequency terms, which are equal to zero in the steady state, i.e., when $V_{1}=\hat{V}_{1}$ and $\theta_{1}=\hat{\theta}_{1}$. However, the dynamics of these terms adversely affect the EPLL small-signal stability [17]. This fact suggests that the EPLL small-signal stability may be improved by mitigating the adverse effects of these terms. To this end, as shown in Fig. 2, adding some double-frequency terms to the EPLL structure is suggested. This modification has been inspired from the adaptive notch filter structure [18]. Note that the signal $\Delta \dot{\hat{\omega}}$, and therefore, the highlighted double-frequency terms in the MsEPLL structure, are equal to zero in the steady

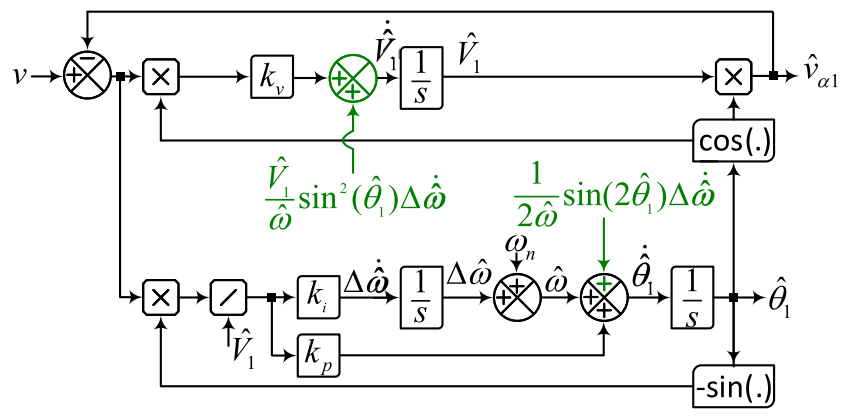

Fig. 2. More-stable EPLL (MsEPLL).

state. ${ }^{2}$ Note also that the double-frequency term $\frac{1}{2} \sin \left(2 \hat{\theta}_{1}\right)$ in Fig. 2 is equal to $\sin \left(\hat{\theta}_{1}\right) \cos \left(\hat{\theta}_{1}\right)$. Therefore, implementing the MsEPLL does not involve the calculation of additional trigonometric functions.

\section{A. LTP Modeling}

Considering (1) as the input signal of the MsEPLL in Fig. 2, its GNDEs can be obtained as

$$
\begin{aligned}
\Delta \dot{\hat{\omega}}= & \frac{k_{i}}{2 \hat{V}_{1}}\left[V_{1} \sin \left(\theta_{1}-\hat{\theta}_{1}\right)+\hat{V}_{1} \sin \left(2 \hat{\theta}_{1}\right)-V_{1} \sin \left(\theta_{1}+\hat{\theta}_{1}\right)\right] \\
\dot{\hat{\theta}}_{1}= & \hat{\omega}+\frac{k_{p}}{k_{i}} \Delta \dot{\hat{\omega}}+\frac{1}{2 \hat{\omega}} \sin \left(2 \hat{\theta}_{1}\right) \Delta \dot{\hat{\omega}} \\
\dot{\hat{V}}_{1}= & \frac{k_{v}}{2}\left[V_{1} \cos \left(\theta_{1}-\hat{\theta}_{1}\right)-\hat{V}_{1}+V_{1} \cos \left(\theta_{1}+\hat{\theta}_{1}\right)-\hat{V}_{1} \cos \left(2 \hat{\theta}_{1}\right)\right] \\
& +\frac{\hat{V}_{1}}{\hat{\omega}} \sin ^{2}\left(\hat{\theta}_{1}\right) \Delta \dot{\hat{\omega}} .
\end{aligned}
$$

Considering the definitions made in (8), in which each parameter is defined as a nominal value (indicated by the subscript $n$ ) plus a small perturbation (indicated by $\Delta$ ), (5) can be linearized as shown in (9) at the bottom of this page

$$
\begin{aligned}
& V_{1}=V_{n}+\Delta V_{1}, \theta_{1}=\theta_{n}+\Delta \theta_{1}, \omega=\omega_{n}+\Delta \omega \\
& \hat{V}_{1}=V_{n}+\Delta \hat{V}_{1}, \hat{\theta}_{1}=\theta_{n}+\Delta \hat{\theta}_{1}, \hat{\omega}=\omega_{n}+\Delta \hat{\omega} .
\end{aligned}
$$

Note that $\theta_{n}=\int \omega_{n} d t=\omega_{n} t+\varphi_{n}$. Without loss of generality, $\varphi_{n}=0$ is assumed in this article.

\footnotetext{
${ }^{2} \mathrm{~A}$ hidden assumption here is that the single-phase input signal is without harmonics. Note that in the presence of harmonics, the signal $\Delta \dot{\hat{\omega}}$ may contain some oscillatory ripples depending on the EPLL bandwidth.
}

$$
\begin{aligned}
& \Delta \dot{\hat{\omega}}=\overbrace{\frac{k_{i}}{2\left(V_{n}+\Delta \hat{V}_{1}\right)}}^{\approx \frac{k_{i}}{2 V_{n}}\left(1-\frac{\Delta \hat{V}_{1}}{V_{n}}\right)}[\overbrace{\left(V_{n}+\Delta V_{1}\right) \sin \left(\Delta \theta_{1}-\Delta \hat{\theta}_{1}\right)}^{\approx V_{n}\left(\Delta \theta_{1}-\Delta \hat{\theta}_{1}\right)}+\overbrace{\left(V_{n}+\Delta \hat{V}_{1}\right) \sin \left(2 \theta_{n}+2 \Delta \hat{\theta}_{1}\right)-\left(V_{n}+\Delta V_{1}\right) \sin \left(2 \theta_{n}+\Delta \theta_{1}+\Delta \hat{\theta}_{1}\right)}^{\approx-V_{n}\left(\Delta \theta_{1}-\Delta \hat{\theta}_{1}\right) \cos \left(2 \theta_{n}\right)-\left(\Delta V_{1}-\Delta \hat{V}_{1}\right) \sin \left(2 \theta_{n}\right)}] \\
& \approx \frac{k_{i}}{2}[\left(1-\cos \left(2 \theta_{n}\right)\right) \overbrace{\left(\Delta \theta_{1}-\Delta \hat{\theta}_{1}\right)}^{\Delta \theta_{e}}-\sin \left(2 \theta_{n}\right) \overbrace{\left(\Delta V_{1}-\Delta \hat{V}_{1}\right)}^{\Delta V_{e}} / V_{n}]
\end{aligned}
$$




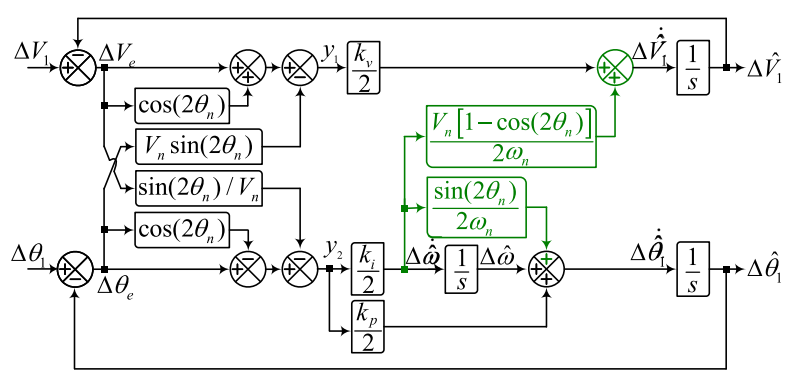

(a)

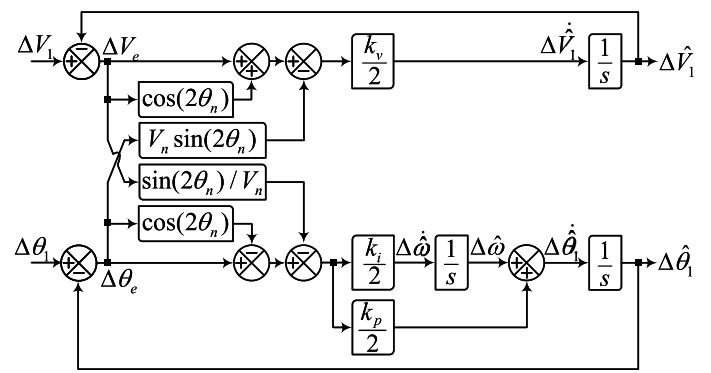

(b)

Fig. 3. (a) LTP model of the MsEPLL. (b) LTP model of the EPLL.

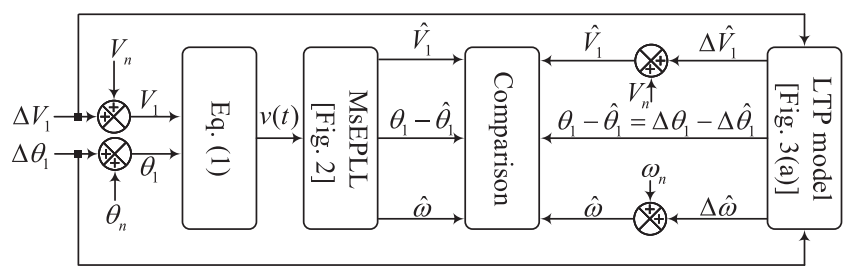

Fig. 4. Model verification procedure.

In a similar manner, one can obtain (10) and (11) by substituting (8) into (6) and (7) and linearizing them

$$
\begin{aligned}
\Delta \dot{\hat{\theta}}_{1} \approx & \Delta \hat{\omega}+\frac{k_{p}}{k_{i}} \Delta \dot{\hat{\omega}}+\frac{1}{2 \omega_{n}} \sin \left(2 \theta_{n}\right) \Delta \dot{\hat{\omega}} \\
\Delta \dot{\hat{V}}_{1} \approx & \frac{k_{v}}{2}\left[\left\{1+\cos \left(2 \theta_{n}\right)\right\} \Delta V_{e}-V_{n} \sin \left(2 \theta_{n}\right) \Delta \theta_{e}\right] \\
& +\frac{V_{n}}{\omega_{n}} \frac{1-\cos \left(2 \theta_{n}\right)}{2} \Delta \dot{\hat{\omega}} .
\end{aligned}
$$

Using (9)-(11), which are a set of LTP differential equations in the time domain, the LTP model of the MsEPLL can be obtained as depicted in Fig. 3(a). Note that the only difference of this model compared to the EPLL LTP model, which is shown in Fig. 3(b) [17], lies in the parts marked with green color. These parts are the result of linearizing the double-frequency terms (green-color terms) in Fig. 2.

To evaluate the accuracy of the obtained LTP model, a timedomain comparison between the MsEPLL and its LTP model as shown in Fig. 4 is carried out. The comparison is conducted in the MATLAB/Simulink. The control parameters of the MsEPLL and its LTP model are considered as $k_{p}=k_{v}=444$ and $k_{i}=49348$ [17]. A small voltage sag test and a small phase jump test are considered for the comparison here. The results of these tests can be observed in Fig. 5. As shown, the predictions of the LTP model are closely matched to the actual

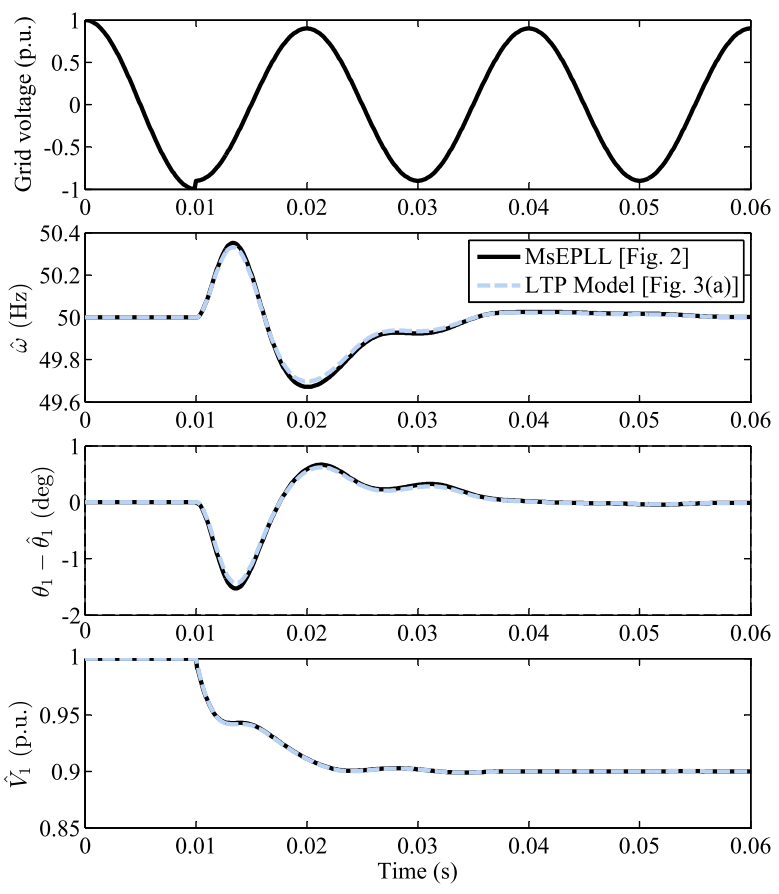

(a)
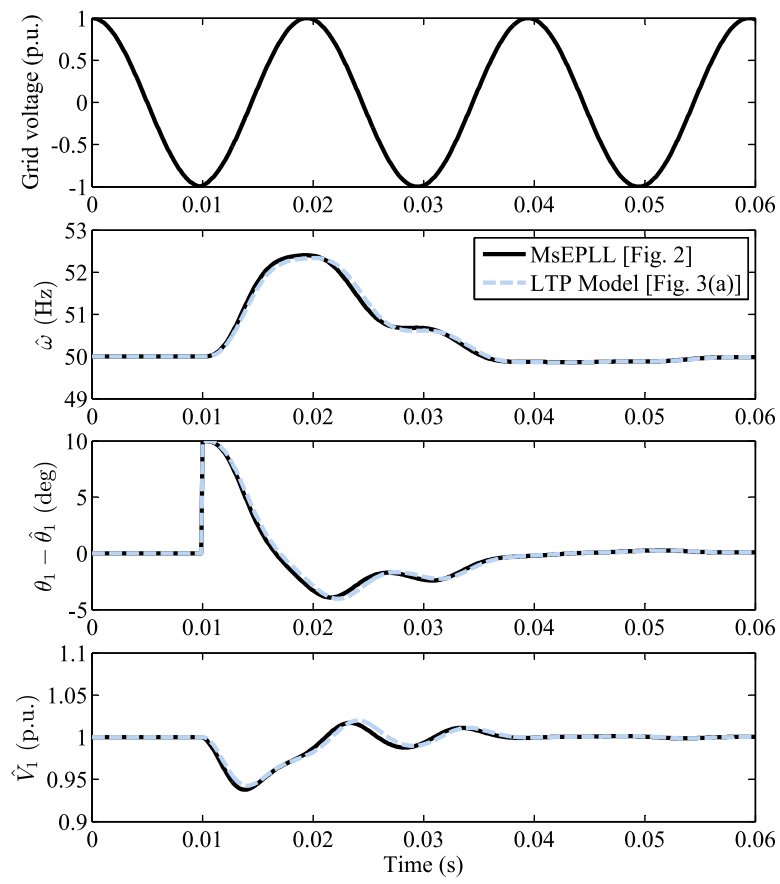

(b)

Fig. 5. Accuracy assessment of the LTP model of the MsEPLL. (a) 0.1-p.u. voltage sag test. (b) $10^{\circ}$ phase jump test.

results. Note that changing the type of the test (for example, considering a frequency jump instead of the voltage sag or phase jump) does not considerably affect the accuracy of the LTP model. However, the magnitude of the disturbance may have a considerable impact on accuracy. For example, if a 0.5-p.u. voltage sag instead of the 0.1-p.u. voltage sag is applied, the model demonstrates a reduced accuracy. The reason is that obtaining the LTP model of the MsEPLL (or, generally, any 
nonlinear system) involves assuming small-signal perturbations around its working points/trajectories. Applying a large-signal disturbance contradicts this assumption and, therefore, causes some inaccuracies.

\section{B. Open-Loop Harmonic Transfer Function (HTF)}

To facilitate the understanding of the procedure for obtaining the open-loop HTF of the MsEPLL, consider the signal $y_{1}$ in Fig. 3(a). In the time domain, this signal is equal to

$$
y_{1}(t)=\left(1+\cos \left(2 \theta_{n}\right)\right) \Delta V_{e}(t)-V_{n} \sin \left(2 \theta_{n}\right) \Delta \theta_{e}(t) .
$$

Replacing the trigonometric functions in (12) by their equivalent expressions in terms of exponential, i.e., $\cos \left(2 \theta_{n}\right)=$ $0.5\left(e^{j \omega_{p} t}+e^{-j \omega_{p} t}\right)$ and $\sin \left(2 \theta_{n}\right)=-j 0.5\left(e^{j \omega_{p} t}-e^{-j \omega_{p} t}\right)$, where $\omega_{p}=2 \omega_{n}$, and applying the Laplace transform results in

$$
\begin{aligned}
y_{1}(s)= & \Delta V_{e}(s)+\frac{1}{2} \Delta V_{e}\left(s-j \omega_{p}\right)+\frac{1}{2} \Delta V_{e}\left(s+j \omega_{p}\right) \\
& -V_{n}\left[\frac{j}{2} \Delta \theta_{e}\left(s+j \omega_{p}\right)-\frac{j}{2} \Delta \theta_{e}\left(s-j \omega_{p}\right)\right] .
\end{aligned}
$$

Substituting $s$ by $s+j m \omega_{p}(m \in \mathbb{Z})$ in (13) and arranging the resulting equations in a matrix form gives (14) shown at the bottom of this page. This matrix equation, which is of infinite dimension, is the HTF of (12). Considering the aforementioned way of formulation, one can easily obtain the open-loop HTF of the MsEPLL as (15) shown at the bottom of this page. Note that some terms of (15) have been already described in (14). The remaining terms are described in (16) shown at the bottom of this page.

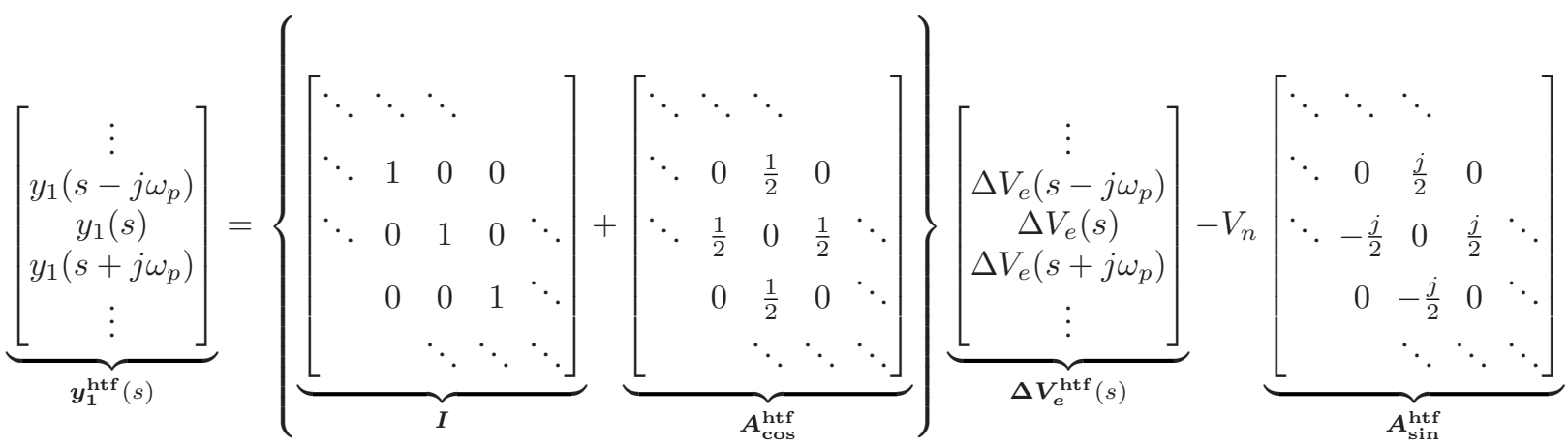

$$
\begin{aligned}
& \underbrace{\left[\begin{array}{c}
\vdots \\
\Delta \theta_{e}\left(s-j \omega_{p}\right) \\
\Delta \theta_{e}(s) \\
\Delta \theta_{e}\left(s+j \omega_{p}\right) \\
\vdots
\end{array}\right]}_{\Delta \boldsymbol{\theta}_{\boldsymbol{e}}^{\text {htf }}(s)}
\end{aligned}
$$

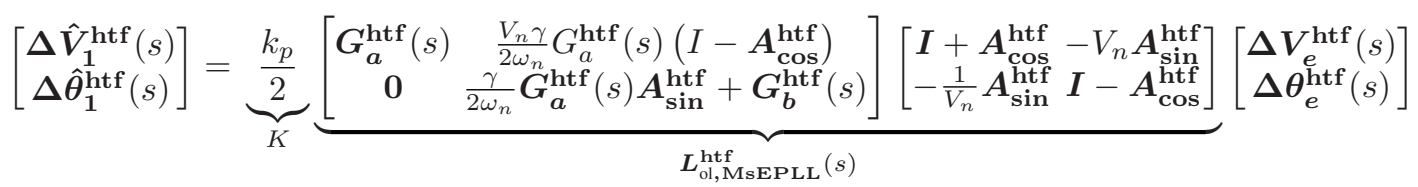

$$
\begin{aligned}
& \gamma=\frac{k_{i}}{k_{p}}, G_{a}(s)=\frac{1}{s}, G_{b}(s)=\frac{s+\gamma}{s^{2}} \\
& \boldsymbol{G}_{\boldsymbol{a}}^{\mathbf{h t f}}(s)=\operatorname{diag}\left(\cdots, G_{a}\left(s-j \omega_{p}\right), G_{a}(s), G_{a}\left(s+j \omega_{p}\right), \cdots\right) \\
& G_{\boldsymbol{b}}^{\mathrm{htf}}(s)=\operatorname{diag}\left(\cdots, G_{b}\left(s-j \omega_{p}\right), G_{b}(s), G_{b}\left(s+j \omega_{p}\right), \cdots\right) \\
& \boldsymbol{\Delta} \hat{\boldsymbol{\theta}}_{\mathbf{1}}^{\mathbf{h t f}}(s)=\left[\cdots, \Delta \hat{\theta}_{1}\left(s-j \omega_{p}\right), \Delta \hat{\theta}_{1}(s), \Delta \hat{\theta}_{1}\left(s+j \omega_{p}\right), \cdots\right]^{T} \\
& \Delta \hat{\boldsymbol{V}}_{\mathbf{1}}^{\mathbf{h t f}}(s)=\left[\cdots, \Delta \hat{V}_{1}\left(s-j \omega_{p}\right), \Delta \hat{V}_{1}(s), \Delta \hat{V}_{1}\left(s+j \omega_{p}\right), \cdots\right]^{T}
\end{aligned}
$$

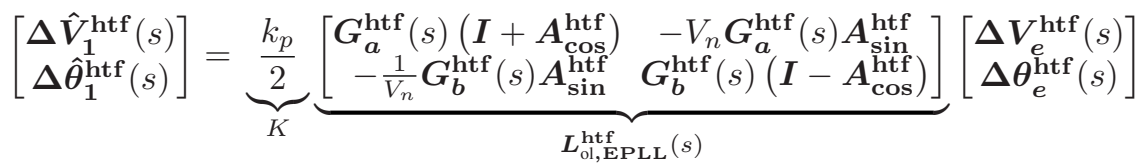




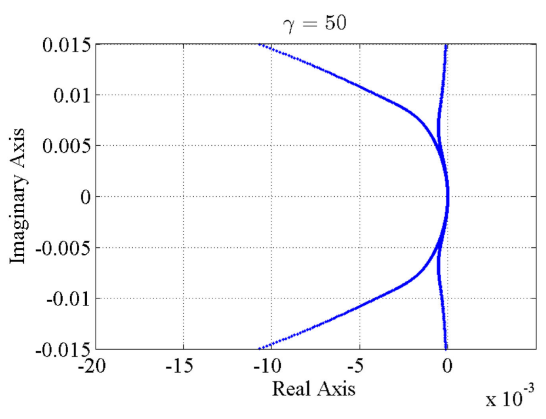

(a)

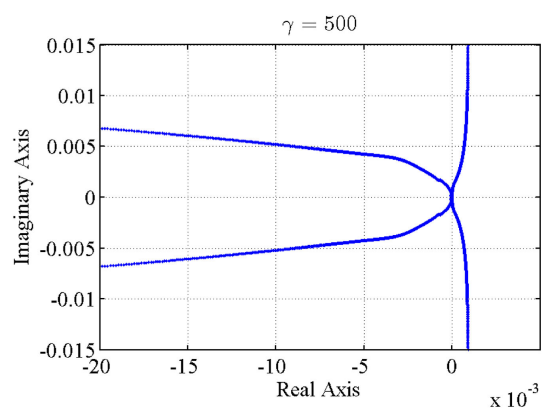

(b)

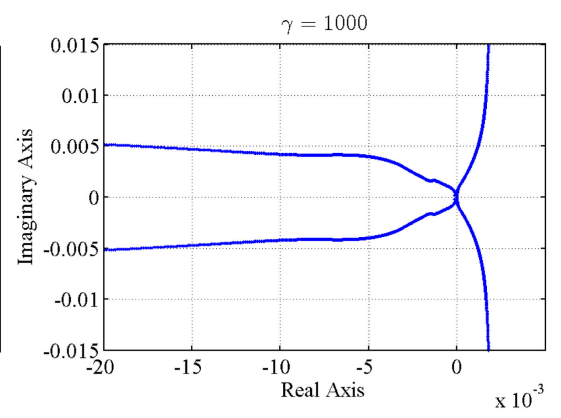

(c)

Fig. 6. Eigenloci of the transfer matrix $\boldsymbol{L}_{\mathrm{ol}, \mathrm{MsEPLL}}^{\mathrm{htf}}(s)$ for different value of $\gamma$. (a) $\gamma=50$. (b) $\gamma=500$. (c) $\gamma=1000$.

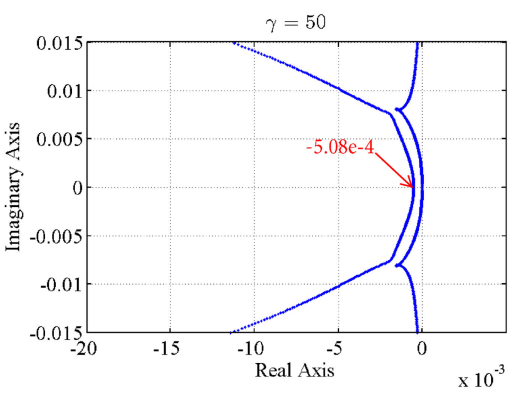

(a)

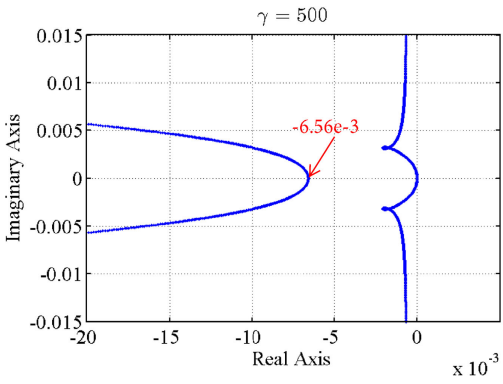

(b)

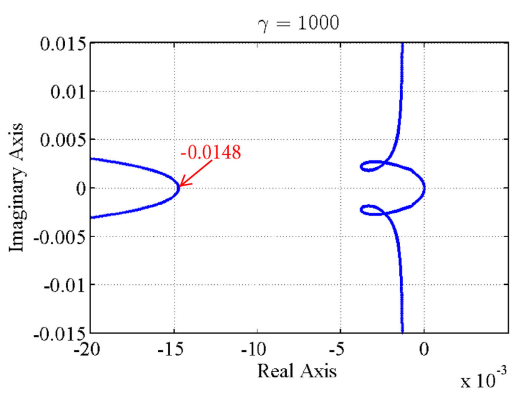

(c)

Fig. 7. Eigenloci of the transfer matrix $\boldsymbol{L}_{\mathrm{ol}, \mathbf{E P L L}}^{\text {htf }}(s)$ for different value of $\gamma$. (a) $\gamma=50$. (b) $\gamma=500$. (c) $\gamma=1000$.

Following a similar procedure as aforementioned, the openloop HTF of the EPLL may also be obtained from its LTP model [see Fig. 3(b)] as shown in (17) shown at the bottom of the previous page.

\section{COMPARISON OF EPLL AND MSEPLL}

\section{A. Stability Region}

Transfer function $\boldsymbol{G}_{\mathrm{ol}, \mathrm{MsEPLL}}^{\mathrm{htf}}(s)=\overbrace{\left(k_{p} / 2\right)}^{K} \boldsymbol{L}_{\mathrm{ol}, \mathrm{MsEPLL}}^{\mathrm{htf}}(s)$ in (15), as mentioned before, is the open-loop HTF of the MsEPLL. According to the generalized LTP Nyqusit stability criterion, the small-signal stability of the MsEPLL can be assessed by obtaining the open-loop eigenloci of $\boldsymbol{L}_{\text {ol, MsEPLL }}^{\text {htf }}(s)$ for $s$ belonging to a strip defined by $-j \omega_{p} / 2<\operatorname{Im}(s)<j \omega_{p} / 2$ and counting the number of encirclement of the point $-1 / K+j 00^{3}$

Fig. 6 shows the eigenloci of the transfer matrix $\boldsymbol{L}_{\mathrm{ol}, \mathrm{MsEPLL}}^{\mathrm{htf}}(s)$ for different value of $\gamma$, which is the only control parameter in this transfer matrix. It is observed that by increasing the value of $\gamma$, an eigenvalue curve of $\boldsymbol{L}_{\mathrm{ol}, \mathrm{MsEPLL}}^{\mathrm{htf}}(s)$ gets closer to the negative real axis. However, regardless of the value of $\gamma$, it does not intersect the negative real axis, and therefore, cannot encircle the point $-1 / K+j 0$ for the positive $K$. Considering that $\boldsymbol{L}_{\mathrm{ol}, \mathrm{MsEPLL}}^{\mathrm{htf}}(s)$ has no unstable pole, it can be concluded that the MsEPLL is stable for positive $K=k_{p} / 2$, and therefore, positive $k_{p}$. The same observation and stability conclusion can be made for any other value of $\gamma$. It means that the MsEPLL is stable in the positive parameter space, i.e., $k_{p}>0$ and $k_{i}>0$, from a small-signal point of view.

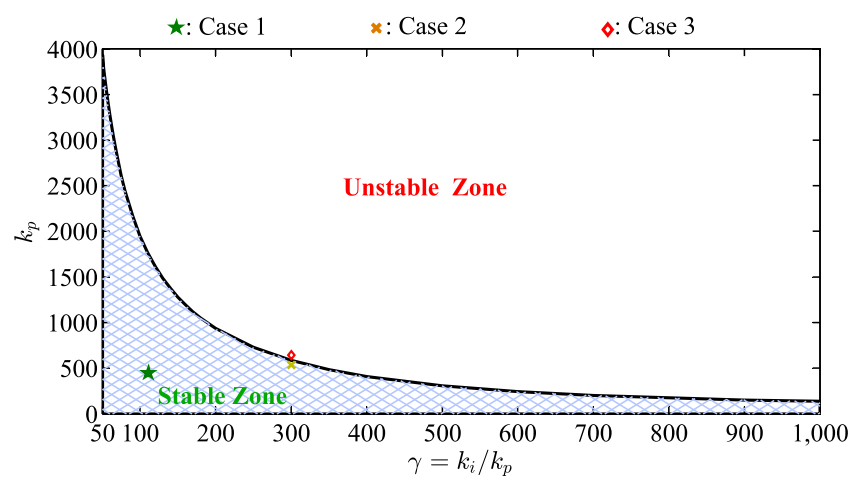

Fig. 8. Small-signal stability region of the EPLL.

For the case of the EPLL, however, the situation is different [17]. This fact can be better visualized in Fig. 7, which shows the eigenloci of the transfer matrix $\boldsymbol{L}_{\mathrm{ol}, \mathrm{EPLL}}^{\mathrm{htf}}(s)$ for different value of $\gamma$. For instance, in Fig. 7(a), which corresponds to $\gamma=50$, an eigenvalue curve of $\boldsymbol{L}_{\mathrm{ol}, \mathbf{E P L L}}^{\mathrm{htf}}(s)$ intersects the negative real axis at $-5.08 e-4$. According to the generalized LTP Nyqusit stability criterion, it means that the EPLL, which its open-loop HTF is $\boldsymbol{G}_{\mathrm{ol}, \mathbf{E P L L}}^{\mathrm{htf}}(s)=K \boldsymbol{L}_{\mathrm{ol}, \mathbf{E P L L}}^{\mathrm{htf}}(s)$, remains stable if $K=\frac{k_{p}}{2}<\frac{1}{5.08 e-4}=1968.5$ or, equivalently, $k_{p}<3937$. Similarly, according to Fig. 7(b) and (c), which are corresponding to $\gamma=500$ and $\gamma=1000$, respectively, it can be concluded

\footnotetext{
${ }^{3} \boldsymbol{L}_{\mathrm{ol}, \mathrm{MsEPLL}}^{\mathrm{htf}}(s)$ is of infinite dimension. Therefore, a truncated version of it should be considered for the stability analysis.
} 


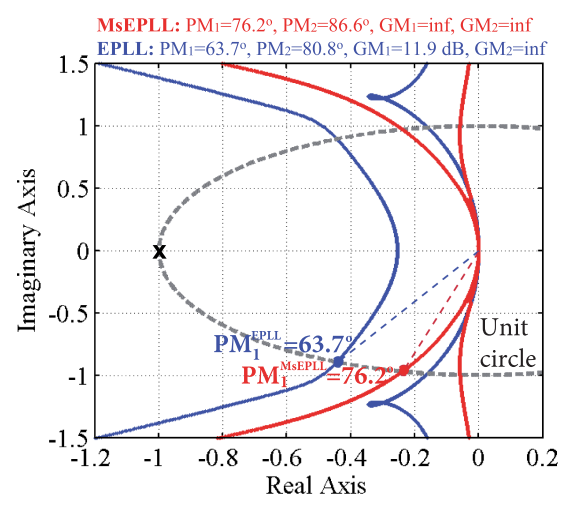

(a)

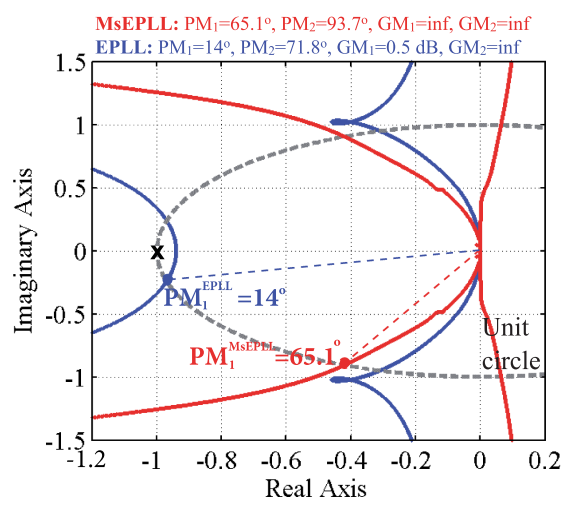

(b)

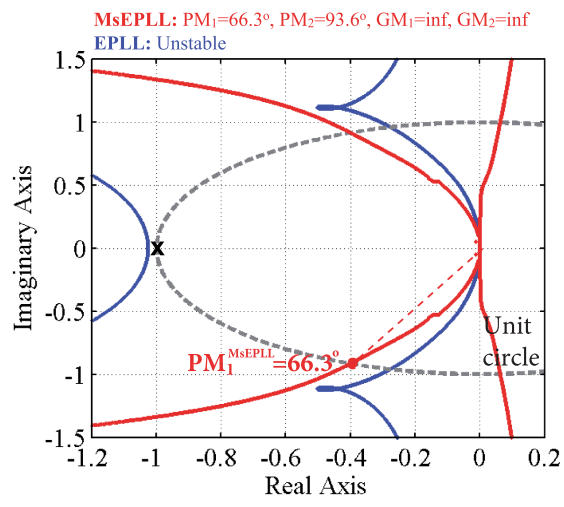

(c)

Fig. 9. Open-loop LTP Nyquist plots of the EPLL and MsEPLL for different sets of control parameters. (a) Case 1. (b) Case 2. (c) Case 3. In all cases, to avoid confusion, only one eigenvalue PM of the EPLL and MsEPLL is highlighted.

that the EPLL remains stable if $K=\frac{k_{p}}{2}<\frac{1}{6.56 e-3}=152.4$ and $K=\frac{k_{p}}{2}<\frac{1}{0.0148}=67.6$ or, equivalently, $k_{p}<304.9$ and $k_{p}<135.1$. It is clear that by increasing the value of $\gamma$, the stable range of $k_{p}$ is decreased. Fig. 8 , which shows the stability region of the EPLL when $\gamma=k_{i} / k_{p}$ changes in the range of 50 to 1000 , helps to better visualize this fact.

In summary, the MsEPLL is unconditionally stable in the positive parameter space from a small-signal point of view. The EPLL, however, has a considerable unstable zone.

\section{B. Stability Margin}

The main aim of this section is to conduct a comparison between the EPLL and MsEPLL from the stability margin point of view. Three case studies, which are corresponding to three sets of control parameters, are considered.

1) Case 1: In this case, the control parameters of the EPLL and MsEPLL are considered as $k_{p}=k_{v}=444$ and $\gamma=$ $k_{i} / k_{p}=111.14$ [17]. This set of parameters, which is marked with a green star in Fig. 8, is in the stable zone of the EPLL and away from its stability border.

Fig. 9(a) shows the open-loop LTP Nyquist plots of the EPLL and MsEPLL for Case 1. Note that the open-loop eigenloci of both the EPLL and MsEPLL intersect the lower half of the unit circle and also the real axis at two points. It means that they have two eigenvalue phase margins (PMs) and two eigenvalue gain margins (GMs). The eigenvalue PMs and GMs of the EPLL and MsEPLL, which indicate how close their open-loop eigenloci are to the critical point $-1+j 0$, can be observed in Fig. 9(a). It is observed that the MsEPLL has a rather more decent stability margin than the EPLL.

To verify the aforementioned observation, a comparison between the EPLL and MsEPLL in response to a phase jump test is carried out. The results of this test can be seen in Fig. 10. It can be observed that the MsEPLL has a lower overshoot in estimating the phase, frequency, and amplitude and, therefore, has a more damped dynamic response. For instance, the overshoot of the MsEPLL in estimating the phase angle is 38\%, while it is around $50 \%$ for the case of the EPLL. Note that the PM of a control system and its overshoot in response to a step input are closely
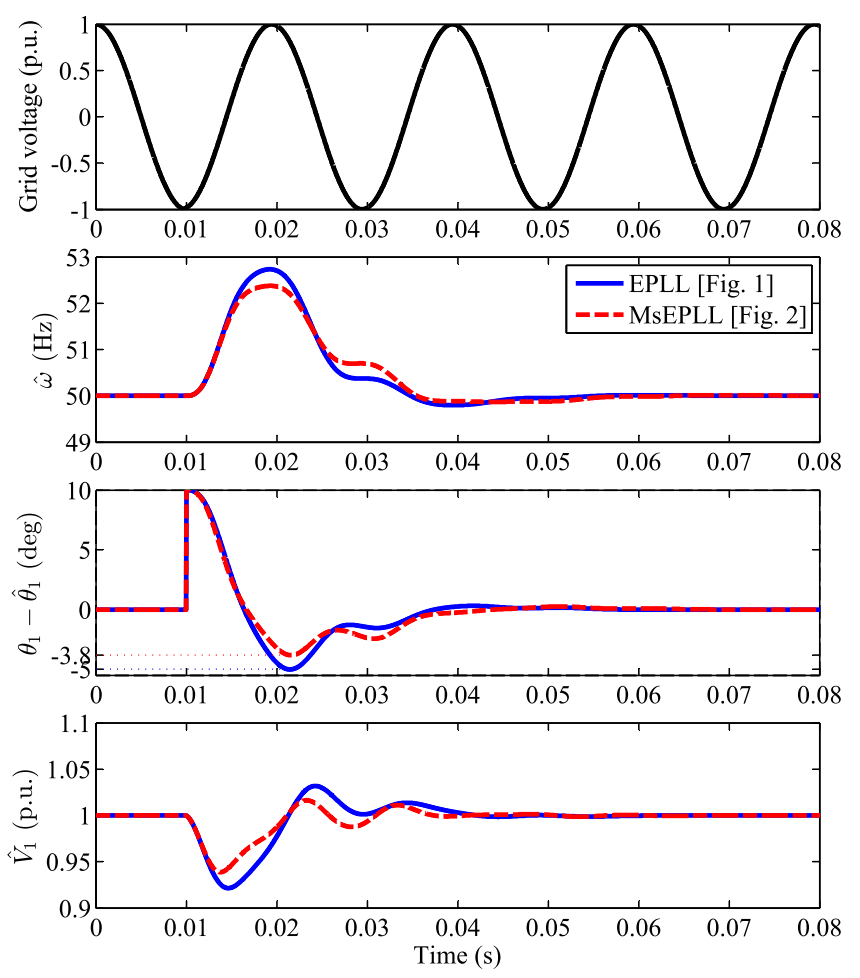

Fig. 10. Comparison between the EPLL and MsEPLL under Case $1\left(k_{p}=\right.$ $k_{v}=444$ and $\left.\gamma=k_{i} / k_{p}=111.14\right)$ and in response to $10^{\circ}$ phase jump test.

related to each other; a lower PM always results in a larger overshoot [19].

2) Case 2: In this case, the control parameters of the EPLL and MsEPLL are considered as $k_{p}=k_{v}=550$ and $\gamma=$ $k_{i} / k_{p}=300$. This set of parameters, which is marked with a $\times$ character in Fig. 8, is in the stable zone of the EPLL, but close to the stability border.

Fig. 9(b) shows the open-loop LTP Nyquist plots of the EPLL and MsEPLL and highlights their eigenvalue PMs and GMs. It is observed that an open-lop eigenvalue curve of the EPLL is close to the critical point $-1+j 0$, and therefore, causes a low PM and GM. Such a low PM is expected to cause a highly 

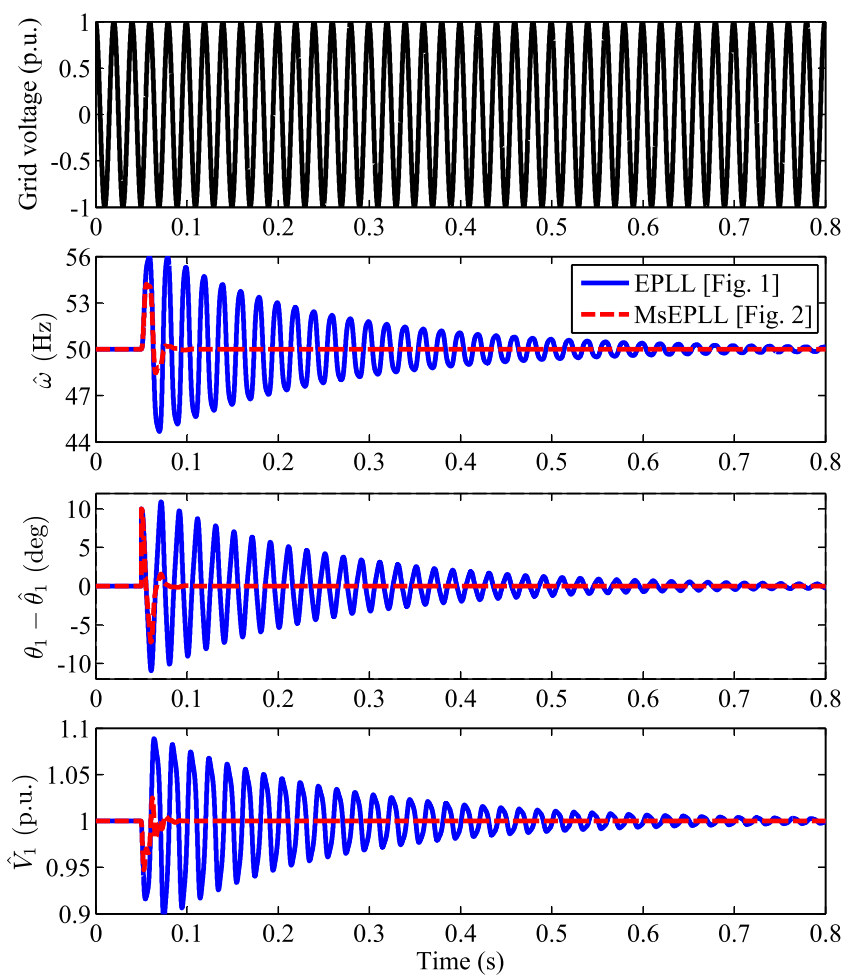

Fig. 11. Comparison between the EPLL and MsEPLL in response to $10^{\circ}$ phase jump test under Case $2\left(k_{p}=k_{v}=550\right.$ and $\left.\gamma=k_{i} / k_{p}=300\right)$.
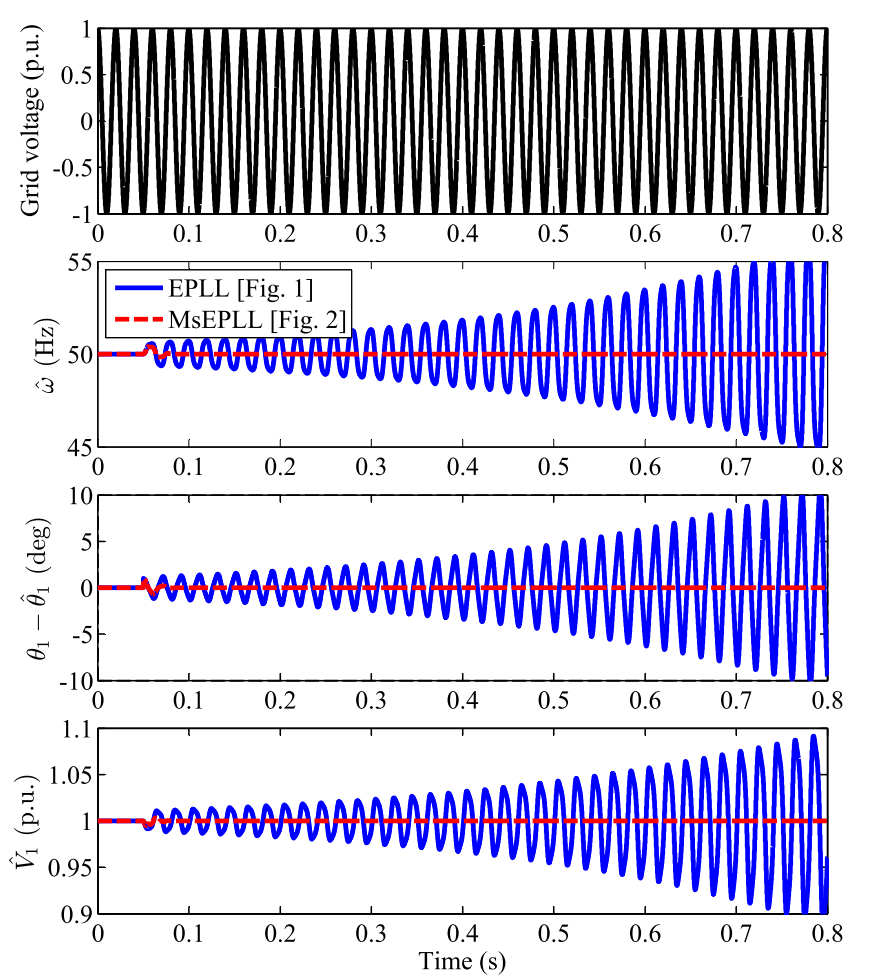

Fig. 12. Comparison between the EPLL and MsEPLL in response to $1^{\circ}$ phase jump. Before the phase jump, the control parameters are set to the values selected for Case 2. At the same moment with the phase jump, the control parameters are changed to the values selected for Case 3 .
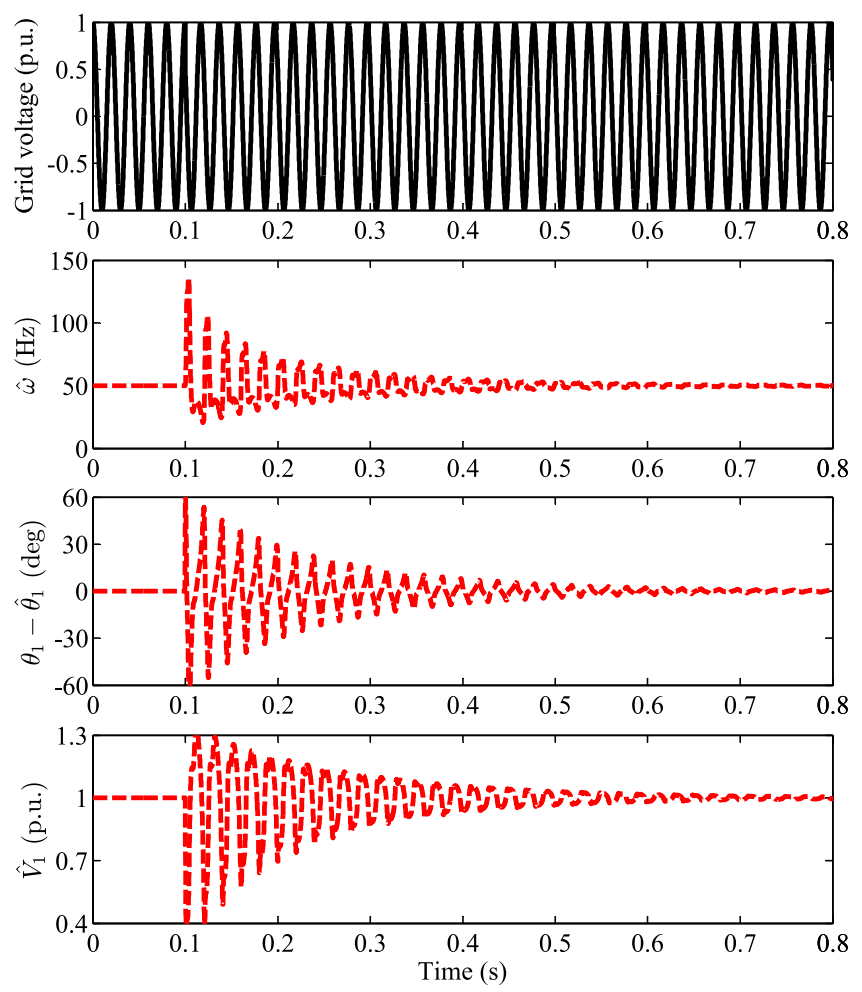

Fig. 13. Performance of the MsEPLL in response to a $60^{\circ}$ phase jump. The selected control parameters are $k_{p}=k_{v}=4000$ and $\gamma=k_{i} / k_{p}=1000$. Note that the results of the EPLL are not shown as it is unstable in this case.

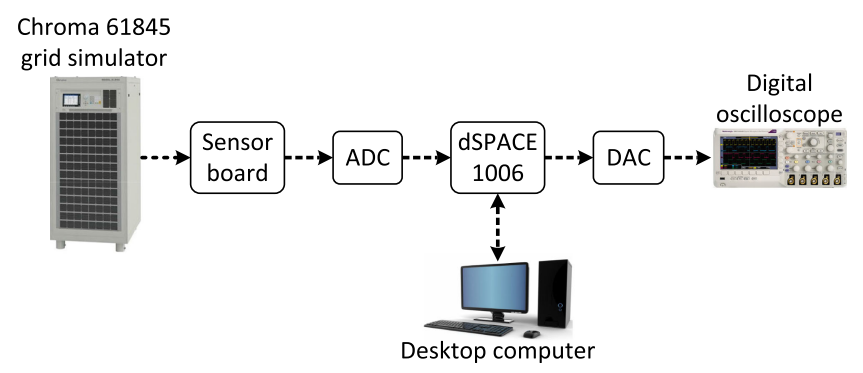

Fig. 14. Experimental setup.

oscillatory dynamic response for the EPLL. The MsEPLL, however, has a quite decent stability margin.

To verify the aforementioned observation, the comparison of the EPLL and MsEPLL in response to the $10^{\circ}$ phase jump test is repeated. The obtained results are shown in Fig. 11. As expected, the transient response of the EPLL is highly oscillatory. The MsEPLL, however, has a much more damped dynamic response and reaches the steady state in a short while after the phase jump.

3) Case 3: In this case, $k_{p}=k_{v}=600$ and $\gamma=k_{i} / k_{p}=$ 300 are selected. This set of parameters, which is marked with a red diamond in Fig. 8, is in the unstable zone of the EPLL, but close to the stability border.

Fig. 9(c) shows the open-loop LTP Nyquist plots of the EPLL and MsEPLL. As shown, an eigenvalue curve of the EPLL encircles the critical point $-1+j 0$, which makes it unstable. The MsEPLL, however, has a decent stability margin.

To verify the aforementioned observations, the control parameters of the EPLL and MsEPLL are initially set to the 

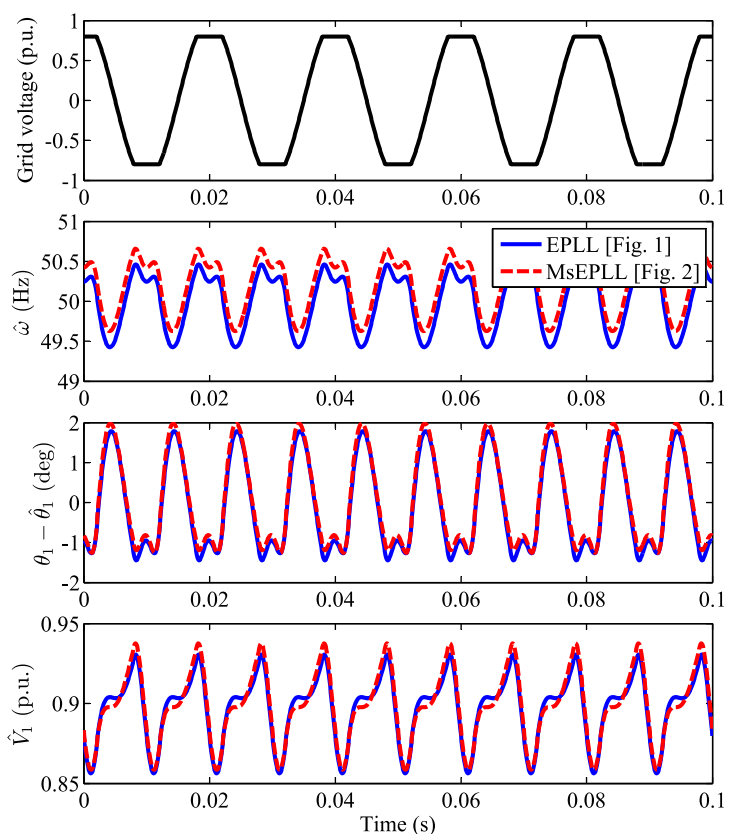

(a)

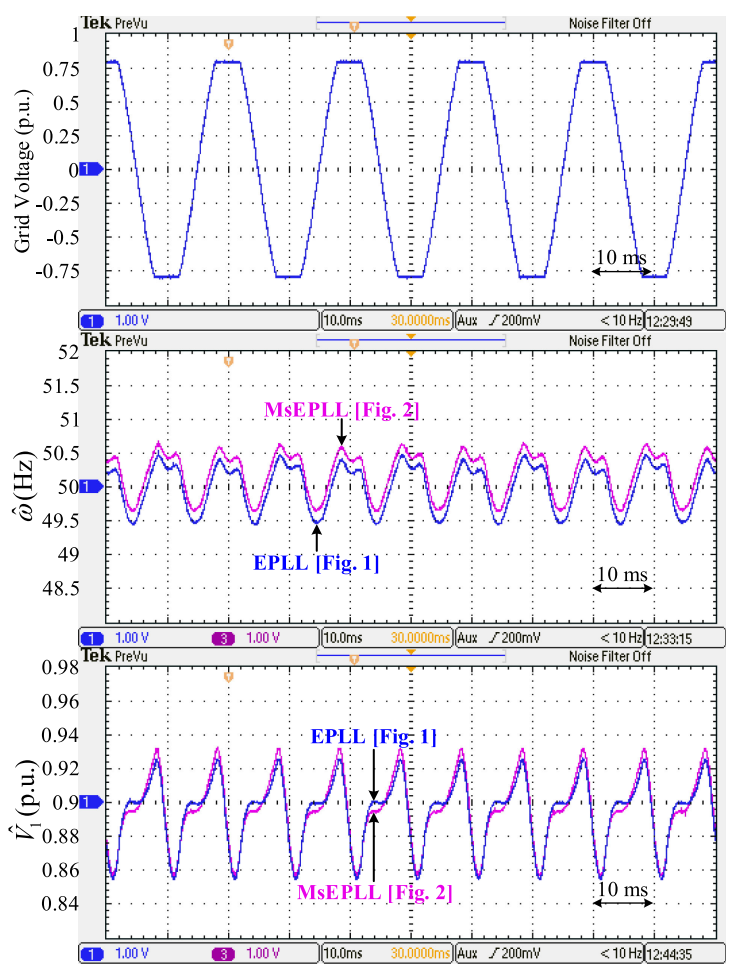

(b)

Fig. 15. Comparison between the EPLL and MsEPLL under a harmonic-rich grid condition. (a) Simulation results. (b) Experimental results. Note that the phase error signal in the experimental results cannot be shown as the actual phase angle is unknown.

values selected for Case 2. Suddenly, the control parameters are changed to the values selected for Case 3 . At the same time, an extremely small phase jump ( $1^{\circ}$ phase jump) is also applied. The result of this test can be observed in Fig. 12. As expected the EPLL becomes unstable. The MsEPLL, however, remains stable.
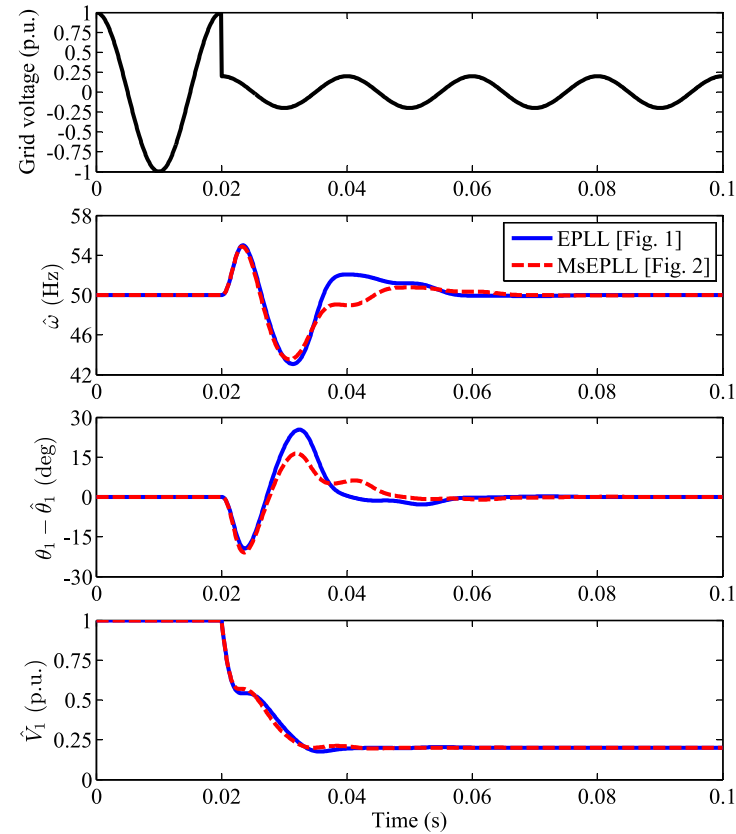

(a)

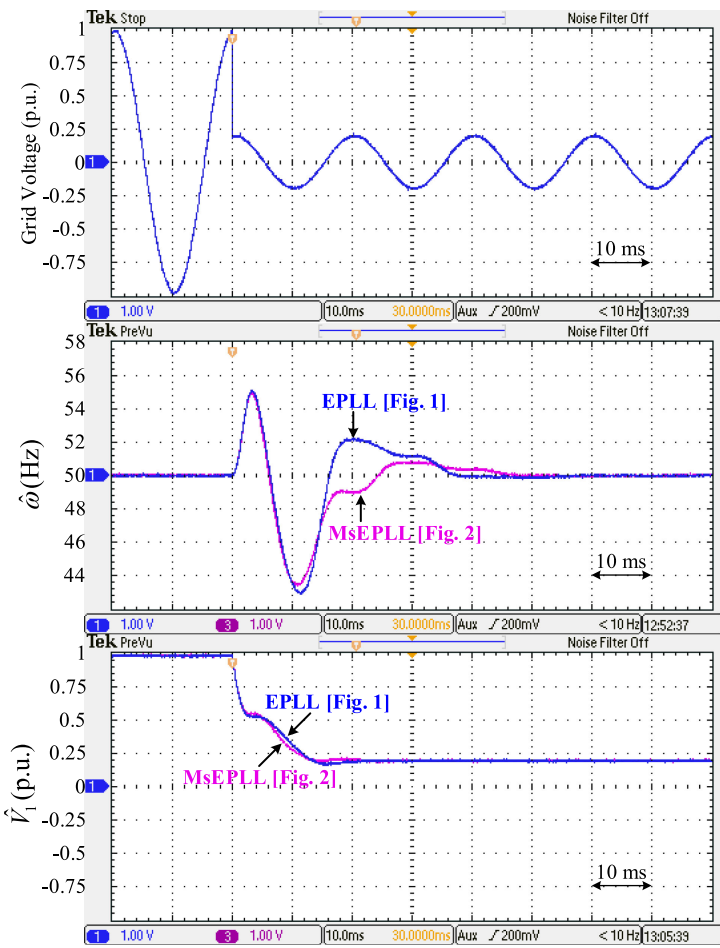

(b)

Fig. 16. Comparison between the EPLL and MsEPLL under a 0.8-p.u. voltage sag. (a) Simulation results. (b) Experimental results. Note that the phase error signal in the experimental results cannot be shown as the actual phase angle is unknown.

By obtaining its open-loop eigenloci or using time-domain simulation results, it can be shown that the MsEPLL is stable at any point in the unstable zone of the EPLL in Fig. 8. For instance, Fig. 13, which shows the time-domain simulation results of the MsEPLL in response to a large-signal test ( $60^{\circ}$ phase jump) supports this fact. Note that the selected control parameters, i.e., 
$k_{p}=k_{v}=4000$ and $\gamma=k_{i} / k_{p}=1000$, are corresponding to the upper right-hand corner of the positive parameter space in Fig. 8. Note also that the results of the EPLL are not shown as it is unstable.

\section{Further Investigations}

In this section, further comparative investigations between the EPLL and MsEPLL are conducted. Throughout these studies, the control parameters of the EPLL and MsEPLL are set to the same values selected for Case 1 .

First, it can be interesting to see whether the enhanced stability margin of the MsEPLL compared to the EPLL causes a negative effect on its filtering capability. To this end, a comparison between the EPLL and MsEPLL under a harmonically distorted grid condition is carried out. The grid voltage signal is considered as a sinusoid that has been clipped to 0.8 p.u. at both the top and bottom. Note that such a signal is corresponding to a total harmonic distortion (THD) approximately equal to $9 \%$. Both the simulation and experimental results of this test are presented. The experimental setup can be observed in Fig. 14. It can be observed in the obtained results (see Fig. 15) that the EPLL and MsEPLL have a comparable filtering capability. It means that the enhanced stability margin of the MsEPLL compared to the EPLL does not negatively affect its filtering ability.

Considering that voltage sags are a common disturbance in power systems, and $\hat{V}_{1}$ (which is an estimation of the grid voltage amplitude) is a key component in realizing the MsEPLL (see highlighted terms in Fig. 2), a comparison between the EPLL and MsEPLL under a voltage sag scenario can be interesting. To this end and, at the same time, to highly excite their nonlinearities, a severe voltage sag ( 0.8 p.u.) is considered. The simulation and experimental results of this test can be observed in Fig. 16. As shown, not very large difference between the transient behavior of the EPLL and MsEPLL is observed; the MsEPLL just has a bit more damped dynamic response.

\section{CONCLUSION}

Improving the stability properties of the EPLL, which is a versatile signal processing tool in different engineering applications, was the main motivation of this article. To this end, a so-called MsEPLL was presented. By deriving an LTP model for the MsEPLL and obtaining its open-loop HTF, it was demonstrated that the MsEPLL (contrary to the conventional EPLL) is unconditionally stable in the positive parameter space from a small-signal point of view. It was also demonstrated that the MsEPLL always has a more decent stability margin compared to the conventional EPLL. All these observations were verified using some numerical and experimental tests.

In addition to improve the EPLL stability properties, this article may contribute toward improving the stability properties of other single-phase synchronization systems. The reason is that the EPLL, as shown in [3], has a close relationship with a large number of single-phase synchronization systems. Therefore, a similar approach as that presented in this article may be used for improving the stability properties of these single-phase synchronization systems.

\section{ACKNOWLEDGMENT}

The authors would like to thank The DSR and The Villum Foundation for technical support.

\section{REFERENCES}

[1] M. Karimi-Ghartemani, Enhanced Phase-Locked Loop Structures for Power and Energy Applications. New York, NY, USA: Wiley, 2014.

[2] S. Golestan, J. M. Guerrero, and J. C. Vasquez, "Single-phase PLLs: A review of recent advances," IEEE Trans. Power Electron., vol. 32, no. 12, pp. 9013-9030, Dec. 2017.

[3] M. Karimi-Ghartemani, "A unifying approach to single-phase synchronous reference frame PLLs," IEEE Trans. Power Electron., vol. 28 , no. 10 , pp. 4550-4556, Oct. 2013.

[4] M. Karimi-Ghartemani, H. Mokhtari, M. R. Iravani, and M. Sedighy, "A signal processing system for extraction of harmonics and reactive current of single-phase systems," IEEE Trans. Power Del., vol. 19, no. 3, pp. 979-986, Jul. 2004.

[5] M. Karimi-Ghartemani, S. A. Khajehoddin, P. K. Jain, A. Bakhshai, and M. Mojiri, "Addressing DC component in PLL and notch filter algorithms," IEEE Trans. Power Electron., vol. 27, no. 1, pp. 78-86, Jan. 2012.

[6] M. Karimi-Ghartemani, B. T. Ooi, and A. Bakhshai, "Application of enhanced phase-locked loop system to the computation of synchrophasors," IEEE Trans. Power Del., vol. 26, no. 1, pp. 22-32, Jan. 2011.

[7] P. Kumar and G. Gurrala, "IEEE C37.118.1a-2014 compliance testing of EPLL and DFAC-PLL for synchrophasors," in Proc. IEEE North Amer. Power Symp., 2018, pp. 1-6.

[8] Z. R. Talukder, K. Nishat Tasnim, and M. S. Reza, "A comparative study of various methods of phasor measurement unit algorithms," in Proc. IEEE 1st Int. Conf. Adv. Sci., Eng. Robot. Technol., 2019, pp. 1-6.

[9] M. Karimi-Ghartemani and J. A. Walseth, "Using the EPLL algorithm as a preprocessor for fault analysis," in Proc. IEEE 11th Int. Conf. Inf. Sci., Signal Process. Appl., 2012, 2012, pp. 1377-1382.

[10] H. Zamani, M. Karimi-Ghartemani, and M. Mojiri, "Analysis of power system oscillations from PMU data using an EPLL-based approach," IEEE Trans. Instrum. Meas., vol. 67, no. 2, pp. 307-316, Feb. 2018

[11] S. A. Khajehoddin, M. Karimi-Ghartemani, P. K. Jain, and A. Bakhshai, "A resonant controller with high structural robustness for fixed-point digital implementations," IEEE Trans. Power Electron., vol. 27, no. 7, pp. 3352-3362, Jul. 2012.

[12] M. Karimi-Ghartemani, S. A. Khajehoddin, P. K. Jain, and A. Bakhshai, "Problems of startup and phase jumps in PLL systems," IEEE Trans. Power Electron., vol. 27, no. 4, pp. 1830-1838, Apr. 2012.

[13] M. Karimi-Ghartemani, S. A. Khajehoddin, P. K. Jain, and A. Bakhshai, "Derivation and design of in-loop filters in phase-locked loop systems," IEEE Trans., Instrum. Meas., vol. 61, no. 4, pp. 930-940, Apr. 2012.

[14] S. Gude and C. Chu, "Dynamic performance enhancement of single-phase and two-phase enhanced phase-locked loops by using in-loop multiple delayed signal cancellation filters," IEEE Trans. Ind. Appl., vol. 56, no. 1, pp. 740-751, Jan./Feb. 2020.

[15] M. Karimi-Ghartemani, S. A. Khajehoddin, P. Jain, and A. Bakhshai, "Comparison of two methods for addressing DC component in phaselocked loop (PLL) systems," in Proc. IEEE Energy Convers. Congr. Expo., Sep. 2011, pp. 3053-3058.

[16] S. Gude and C. Chu, "Single-phase enhanced phase-locked loops based on multiple delayed signal cancellation filters for micro-grid applications," IEEE Trans. Ind. Appl., vol. 55, no. 6, pp. 7122-7133, Nov./Dec. 2019.

[17] S. Golestan, J. M. Guerrero, J. C. Vasquez, A. M. Abusorrah, and Y Al-Turki, "Standard SOGI-FLL and its close variants: Precise modeling in LTP framework and determining stability region/robustness metrics," IEEE Trans. Power Electron., vol. 36, no. 1, pp. 409-422, Jan. 2021.

[18] M. Mojiri and A. R. Bakhshai, "An adaptive notch filter for frequency estimation of a periodic signal," IEEE Trans. Autom. Control, vol. 49, no. 2, pp. 314-318, Feb. 2004.

[19] L. Harnefors, "Modeling of three-phase dynamic systems using complex transfer functions and transfer matrices," IEEE Trans. Ind. Electron. vol. 54, no. 4, pp. 2239-2248, Aug. 2007. 